\title{
Subfulminant Acute Liver Failure by Albendazole: Case Report
}

\author{
Jose Fabio Freire ${ }^{\mathrm{a}, \mathrm{e}}$, Lopasso Maria Carolina Rocha ${ }^{\mathrm{b}}$, Lopes Gabriel Lima ${ }^{\mathrm{c}}$, \\ Pereira Renner Augusto Raposo ${ }^{\mathrm{d}}$
}

\begin{abstract}
Acute hepatic failure is a rare and dramatic condition of acute loss of hepatocellular function, generally caused by virus, auto-immunity or drugs, either by idiosyncratic reaction or by dose-dependent injury. We present a case of hepatic injury induced by albendazole, an imidazole derivate, in a patient of 21 years old, who developed acute hepatic failure. She was included at the transplantation list, but had a great clinical evolution, with full recovery of the liver function only with support measures, prescinding of that treatment. In spite of this drug class presents with hepatotoxicity is already widely known fact, there are just few cases in the literature in association with hepatic failure.
\end{abstract}

Keywords: Hepatotoxicity; Albendazole; Acute liver failure

\section{Introduction}

Acute liver failure is a clinical syndrome, potentially reversible, characterized by the emergence of hepatic encephalopathy within 8 weeks after the onset of signs and symptoms of an acute hepatitis, in patients without evidence of chronic hepatic disease [1]. It is a challenging clinical emergency, and it involves a pattern of specific clinical symptoms and pathophysiologic responses. This clinical entity has several forms of liver

Manuscript accepted for publication April 16, 2015

anternal Medicine, Universidade Federal de Sao Paulo's Hospital, Sao Paulo, Brazil

bIntensive Care Unit, Hospital Sao Paulo, Sao Paulo, Brazil

${ }^{\mathrm{c} C l i n i c a l}$ Oncology Residency at Hospital Sirio-Libanes, Sao Paulo, Brazil; Former Internal Medicine Resident of Universidade Federal de Sao Paulo's Hospital, Sao Paulo, Brazil

dElectrophysiology, Arrhythmia and Artificial Cardiac Stimulation Residency at Instituto do Coracao, Sao Paulo, Brazil; Former Internal Medicine Resident of Universidade Federal de Sao Paulo's Hospital, Sao Paulo, Brazil

${ }^{\mathrm{e} C}$ Corresponding Author: Jose Fabio Freire, Internal Medicine, Universidade Federal de Sao Paulo's Hospital, Rua Napoleao de Barros, 715, Sao Paulo/SP, CEP 04024-002, Brazil. Email: fabiofreire1@gmail.com

doi: http://dx.doi.org/10.14740/jmc2147w injury, such as cytotoxic and the cytopathic injuries.

Cytotoxic lesions consists of a direct hepatocellular damage by viruses (except $\mathrm{C}$ viruses), drugs, metabolites or toxins; among the most common known associated medications are the analgesics, in particular acetaminophen, antiepileptic, antibiotics, antifungal agents and antihypertensives. On the other hand, the cytopathic injury is mediated by an immune response against the hepatocytes [2].

Acute liver failure is an acute and serious deterioration of hepatocellular function secondary to cytotoxicity, mainly caused by external factors, usually by drugs or even by cholestasis. The current incidence of acute fulminant hepatitis is unknown. There are no data about the incidence of liver failure in Brazil; however, it is known that $2 \%$ of liver transplants are made because of acute liver failure [2].

The diagnosis is based on clinical, epidemiological and laboratory findings. The clinical picture is vast, characterized by prodromal symptoms such as nausea, vomiting, low appetite, abdominal discomfort and even by the famous triad, consisting of jaundice, coagulopathy and encephalopathy. Patients usually have dramatic elevations on liver enzyme levels that often exceed 40 times the normality, besides electrolyte changes, coagulation and hematologic tests [3]. These levels do not seem to correlate with prognosis, but the rapid decline of the liver enzyme values, especially more than $60 \%$ of their values in $24 \mathrm{~h}$, is a signal of liver failure [4].

Treatment of acute liver failure consists of specific measures of support such as hospitalization in intensive care unit (ICU) and treatment of complications, like infections and bleeding. Importantly, liver transplantation should be considered in patients who have poor prognostic factors, such as serum level of factor $\mathrm{V}$, lactate and activated prothrombin time (PT). The Gc-globulin dosage, vitamin D carrier globulin, has also been associated with prognostic correlation and compared to the King's College criteria. The use of APACHE criteria is not specific for these patients, but it can be used for evaluation. The MELD score, which is the model score for patients with end-stage liver disease, is useful for patients with chronic hepatocytic failure, but it does not apply for patients with acute liver failure. In this report, we describe a subfulminant acute liver failure in a 21-year-old patient, secondary to the use of albendazole, an antiparasitic drug with few cases described in medical literature with such toxicity [5]. 
Table 1. Admission Exams From Hospital Regional de Rondonopolis/MT

\begin{tabular}{lcccc}
\hline ALT: 1,284 & AF: 412 & TB: 19.83 & DB: 9.02 & IB: 10.81 \\
AST: 1,916 & GGT: 33 & Albumin: 3.8 & PT: $18.5 \%$ & INR: 6.55 \\
Serology for HAV, HBV, HCV, HIV, toxoplasmosis, CMV, EBV e syphilis: negative & \\
$\begin{array}{l}\text { Complete coagulation tests e platelets count: normal } \\
\text { Ultrasound of the abdomen: normal }\end{array}$ & & \\
\hline
\end{tabular}

Table 2. Admission Exams From Hospital Sao Paulo After 10 Days

CBC: Hb: 13.1; Ht: 38.7; leukocytes: 14,000 cells ( $0 \backslash 69 \backslash 2.3 \backslash 1.1 \backslash 20.3 \backslash 7.1)$; platelets: 218,000

\begin{tabular}{lllll} 
ALT: 554 & TB: 20.42 & DB: 19.24 & IB: 1.18 & Albumin: 2.7 \\
Urea: 13 & Creatinine: 0.69 & PT: $35 \%$ & INR: 2,27 & aPTT: 1.42 \\
Sodium: 138 & Phosphorus: 3.4 & Magnesium: 1.9 & Potassium: 4.2 & Glucose: 69 \\
\hline
\end{tabular}

\section{Case Report}

TRSO, female, 21-year-old, natural and precedent from Rondonopolis/MT, Brazil, with brown skin, was married, cashier, with one child. Patient presented with nausea and vomiting, accompanied by malaise, mucocutaneous jaundice, dark urine and fecal acholia on October 19, 2011. She had never presented such symptoms.

The patient denied previous personal or familial liver diseases, surgeries, allergies or recent travel and no co-morbidities. As a routine medication, the patient used only oral contraceptive for over 3 years and denied use of acetaminophen (there were no reports of new medication use at the time of the symptoms onset). She denied she had intake teas or herbs in that period.

The patient searched for medical attention in the Hospital Regional de Rondonopolis, where it was identified a cholestatic framework secondary to acute hepatitis; local physicians dosed inflammatory liver markers, canalicular enzymes, bilirubin, coagulation tests and dosage of serum proteins; the results demonstrated laboratory signs of severe hepatocellular injury, as it is shown in Table 1.

The patient evolved with rapid clinical and laboratory worsening in 1 week, with leukocytosis with nuclear left shift $(25,400$ cells) and hepatic encephalopathy grade III-IV, when it was introduced intravenous ceftriaxone for empirical treatment of infection. She was promptly transferred to the ICU of the Hospital Sao Paulo on October 29, 2011, with an initial diagnosis of fulminant acute hepatitis, for evaluation and priority indication of liver transplantation. The entrance exams in our service are shown in Table 2.

We used only the prognostic score of King's College (no intake of acetaminophen), because it was not possible to dose the serum factor $\mathrm{V}$ to apply the Clichy criteria, as demonstrated in Table 3. As noted, the patient had some criteria of poor prognosis and even sufficient data to indicate a liver transplantation.

In time the patient was in the ICU, she did not present hemodynamic instability, fever, electrolyte disturbances or impaired renal function. We obtained antibodies for autoimmune hepatitis, but all of them were negative.

The patient showed full recovery of the consciousness level and progressive improvement in liver function tests in the first week of hospitalization in our service, when she was transferred to the Women's Infirmary of Internal Medicine Department on November 5, 2011. The patient began to be accompanied by the gastroenterology and liver transplantation teams.

Gradually the patient improved her cholestatic symptoms and showed normalization of bowel habits, remaining, however, jaundice throughout the hospital stay. We noticed the appearance of papular lesions on the front, chin and back regions (with adjacent xeroderma, scratching signs and crusting), shoulders and upper chest (with several pustular lesions), without signals of secondary infection, similar to those the pa-

Table 3. Liver Transplant Indication Criteria in Acute Liver Failure Without Acetaminophen Intake of King's College Hospital

1) $\mathrm{PT}>100 \mathrm{~s}$ or INR $>6.5$ (regardless of the encephalopathy degree)

2) Or three of the following variables:

Age $<10$ or $>40$ years old

Viral hepatitis (A or B), halothane, pharmacological idiosyncratic reactions

Jaundice length $>7$ days before the onset of encephalopathy

$\mathrm{PT}>50 \mathrm{~s}$ or INR $>3.5$

Serum bilirubinemia $>17.5 \mathrm{mg} / \mathrm{dL}$ 
Table 4. Evolution of Patient's Laboratory Tests During Hospitalization From Hospital Sao Paulo

\begin{tabular}{lllll}
\hline & August 30, 2011 & November 01, 2011 & November 04, 2011 & November 08, 2011 \\
\hline ALT & 410 & 245 & 157 & 115 \\
AST & 150 & 128 & 80 & 95 \\
Albumin & 2.4 & 2.3 & 2.7 & 3.6 \\
TB & 23.03 & 20.56 & 20.15 & 19.20 \\
IB & 4.06 & 2.77 & 1.84 & 9.90 \\
DB & 18.97 & 17.79 & 18.31 & 9.30 \\
PT & $34 \%$ & $42 \%$ & $64 \%$ & $87 \%$ \\
INR & 2.34 & 1.92 & 1.36 & 1.09 \\
Urea & 09 & 29 & 17 & 20 \\
Creatinine & 0.73 & 1.03 & 0.95 & 0.82 \\
Glucose & 95 & 74 & 101 & 90 \\
\hline
\end{tabular}

tient had already presented in a previous pregnancy. An evaluation of dermatology was requested, that suggested as the main diagnostic hypotheses folliculitis by Pityrosporum and acneiform eruption, confirming the first hypothesis through skin biopsy performed in two different sites, with punch technique.

During this period, after anamnestic reassessment, we asked the patient's father to seek for any information that could guide us for more information about the cause of the presented liver failure, since the main causes were practically discarded (like acetaminophen use or medicinal plants, infections, autoimmune diseases); he found in her room empty cards of some medications, which the patient remembered of having used 3 days before the onset of her symptoms, when she sought for medical attention with dyspeptic complaints. The patient ingested one capsule of domperidone $10 \mathrm{mg}$, one capsule of omeprazole $20 \mathrm{mg}$ and six tablets of albendazole $400 \mathrm{mg}$ for empiric treatment of intestinal helminths (one tablet every 12 $\mathrm{h}$ for 3 days). Applying the Roussel Uclaf Causality Assessment score (RUCAM) in this case of acute hepatic injury, we obtained a score of 7 , indicating probable causality.

After full recovery of her general condition, both teams decided to proceed with the hospital discharge with a final diagnose of subfulminant acute liver failure, probably secondary to the use of imidazole derivative. Patient's laboratory evolution during hospitalization in ICU and in the clinical infirmary is presented in Table 4.

After 3 weeks of hospital discharge (on November 21, 2011), the patient attended to the gastroenterology department and performed a routine check in hepatology clinic. She presented in excellent general condition, asymptomatic, with only mild jaundice in sclera (remaining with mixed hyperbilirubinemia), and with all the other liver tests completely normal.

\section{Discussion}

Liver injury-induced by drugs (LIID) is a well-documented condition in the scientific environment and its annual incidence in patients with prescribed medication is around 1 in
10,000 - 100,000 exposed people [6]. It accounts for about $10 \%$ of adverse drug reactions [7].

The LIID is part of the differential diagnosis of liver diseases, and this hypothesis must be strengthened in the exclusion of other etiological factors associated with clinical history. Its presentation spectrum is broad, ranging from small laboratory changes to liver failure. Idiosyncratic LIID with acetaminophen intoxication is the major cause of acute liver failure in the United States and Sweden. It is also the leading cause of withdrawal of drugs that are already in clinical use [8, 9]. We have no accurate epidemiological data in Brazil.

Hepatotoxicity by drugs may occur secondary to two mechanisms: direct or idiosyncratic hepatocellular injury. Direct hepatotoxicity may be predictable, since it is dose-dependent and it has a short time between the exposure and the onset of symptoms. The idiosyncratic agents do not have predictable toxicity or dose-dependence [8].

Liver injury triggered by albendazole is relatively common, including mild-to-moderate changes in liver enzymes, reported in approximately $16 \%$ of patients using this medication [10]. However, liver failure secondary to the use of albendazole is extremely rare, with few reported cases in medical literature $[11,12]$. We did not find case reports of fulminant hepatic failure.

The diagnosis of drug-induced liver injury is a challenge that can be obtained through three methods: 1) expert opinion; 2) probabilistic approach; or 3) using diagnostic scales. The diagnostic scale of the Council for International Organizations of Medical Sciences (CIOMS), also known as the causal Assessment Method or RUCAM, although complex and difficult to apply by doctors who are unfamiliar with induced liver injury by drugs, is often used by hepatologists [13].

In our case, applying the CIOMS or the RUCAM scale, a probable association between the consumption of medication and adverse drug reaction was obtained. The possible score results with these scales are: unlikely (1 - 2 points), possible (3 4 points), probable (5 - 8 points) and highly likely ( $>8$ points). The time relationship between the drug and the onset of the symptoms is very suggestive, and in addition, other secondary 
causes of hepatitis were investigated, including viral hepatitis, autoimmune hepatitis among others, and all were excluded.

The remarkable thing in this case is the unfavorable outcome of the drug-induced hepatitis, evolving with subfulminant liver failure. The patient was listed and prioritized for liver transplantation; however, she developed spontaneous improvement of the liver failure only with supportive measures, before the organ donation was achieved.

Other cases of acute hepatitis secondary to use of albendazole have been reported in the literature, however, presenting with a more benign evolution. The treatment of hepatic echinococcosis with albendazole was reported in two previous studies: the first one coursing with jaundice in only one case report [7], and in the second article with the appearance of hepatocellular failure in seven patients in a case series of 40 patients during the treatment with the imidazole. This condition, however, often requires longer treatment with albendazole [8].

In Brazil, the use of antiparasitic agents is very common, both as a treatment for intestinal parasites and also for prophylaxis, often without medical prescription due to the ease of public access to the drug. Based on the occurrence of some cases already reported in the literature, the investigation of liver disease should include the research of albendazole use and other imidazoles during the anmnesis. This theme also deserves further study to better understand the pathophysiology related to the hepatotoxicity of this agent.

\section{References}

1. Danan G, Benichou C. Causality assessment of adverse reactions to drugs--I. A novel method based on the conclusions of international consensus meetings: application to drug-induced liver injuries. J Clin Epidemiol. 1993;46(11):1323-1330.

2. Bucuvalas J, Yazigi N, Squires RH, Jr. Acute liver failure in children. Clin Liver Dis. 2006;10(1):149-168, vii.

3. Fontana R. Acute liver failure. Sleisenger and Fordtran's gastrointestinal and liver disease 2006.

4. Polson J, Lee WM. AASLD position paper: the management of acute liver failure. Hepatology. 2005;41(5):11791197.

5. Sgro C, Clinard F, Ouazir K, Chanay H, Allard C, Guilleminet $\mathrm{C}$, Lenoir C, et al. Incidence of drug-induced hepatic injuries: a French population-based study. Hepatology. 2002;36(2):451-455

6. Shapiro MA, Lewis JH. Causality assessment of druginduced hepatotoxicity: promises and pitfalls. Clin Liver Dis. 2007;11(3):477-505, v.

7. Guidance for Industry. Drug-Induced Liver Injury: Premarketing Clinical Evaluation, July 2010. <http://www. fda.gov/downloads/Drugs/GuidanceComplianceRegulatoryInformation/Guidances/UCM174090>.

8. Wei G, Bergquist A, Broome U, Lindgren S, Wallerstedt S, Almer S, Sangfelt P, et al. Acute liver failure in Sweden: etiology and outcome. J Intern Med. 2007;262(3):393-401.

9. Choi GY, Yang HW, Cho SH, Kang DW, Go H, Lee WC, Lee YJ, et al. Acute drug-induced hepatitis caused by albendazole. J Korean Med Sci. 2008;23(5):903-905.

10. Amoruso C, Fuoti M, Miceli V, Zito E, Celano MR, De Giorgi A, Nebbia G. [Acute hepatitis as a side effect of albendazole: a pediatric case]. Pediatr Med Chir. 2009;31(6):262-264.

11. Garcia-Cortes M, Stephens C, Lucena MI, FernandezCastaner A, Andrade RJ. Causality assessment methods in drug induced liver injury: strengths and weaknesses. J Hepatol. 2011;55(3):683-691.

12. Jagota SC. Jaundice due to albendazole. Comment on Indian J Gastroenterol. 1988;7(4):245-246.

13. Morris DL, Smith PG. Albendazole in hydatid disease-hepatocellular toxicity. Trans R Soc Trop Med Hyg. 1987;81(2):343-344. 